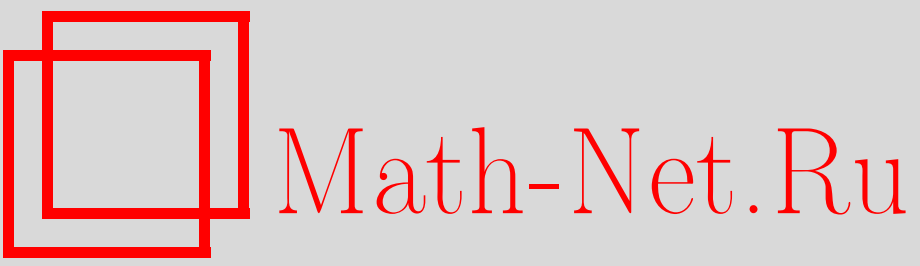

А. Б. Борисов, Асимптотическое поведение сингулярных солитонов и метод обратной задачи рассеяния для решения краевых задач, ТМФ, 2000, том 124, номep 2, 279-291

DOI: https://doi.org/10.4213/tmf639

Использование Общероссийского математического портала Math-Net.Ru подразумевает, что вы прочитали и согласны с пользовательским соглашением

http://www.mathnet.ru/rus/agreement

Параметры загрузки:

IP : 54.157 .27 .8

26 апреля 2023 г., 12:57:34 
ТЕОРЕТИЧЕСКАЯ

И МАТЕМАТИЧЕСКАЯ

ФИЗИКА

Том 124, № 2

август, 2000

(C) 2000 г.

А.Б. Борисов*

\section{АСИМПТОТИЧЕСКОЕ ПОВЕДЕНИЕ СИНГУЛЯРНЫХ СОЛИТОНОВ И МЕТОД ОБРАТНОЙ ЗАДАЧИ РАССЕЯНИЯ ДЛЯ РЕШЕНИЯ КРАЕВЫХ ЗАДАЧ}

В рамках метода обратной задачи рассеяния рассматривается решение краевых задач для уравнения синус-Гордон и эллиптической решетки Тоды. Данный подход позволяет включить в рассмотрение решения в виде сингулярного солитона - спирального вихря.

\section{1. ВВЕДЕНИЕ}

Метод обратной задачи в настоящее время используется как эффективная процедура решения многих универсальных интегрируемых нелинейных уравнений. С его помощью можно получать не только специальные типы решений - многосолитонные и конечнозонные, но и эффективно исследовать асимптотическое поведение многих уравнений гиперболического типа, т.е. исследовать решение задачи Коши так же полно, как это можно сделать в линейном случае. При анализе асимптотического поведения решений на больших временах выяснилось, что несолитонная часть в большинстве случаев ведет себя не так, как решения линеаризованной задачи: нелинейный сдвиг частоты волны приводит к изменению фазы асимптотического решения, и существуют пространственно-временные области с нелинейным характером решения при любой малой амплитуде $[1,2]$.

В последнее время выявились трудности традиционного метода обратной задачи рассеяния при решении начально-краевых задач на полубесконечном интервале. Так при произвольном краевом условии на полубесконечном интервале для нелинейного уравнения Шредингера отображение решений полевых уравнений в данные рассеяния не приводит к линейному уравнению для них, их эволюция определяется нелинейным интегральным уравнением [3]. К настояшему времени методом обратной задачи решались лишь некоторые специальные типы начально-краевых задач [4-6], которые, по-видимому, наиболее эффективно исследовать с помошью преобразования Беклунда или условия совместности краевых условий с высшими симметриями [7]. Наиболее очевидны

* Институт физики металлов УрО РАН, Екатеринбург, Россия. E-mail: Borisov@imp.uran.ru 
трудности решения подобных задач в случае дискретных систем, которые в континуальном пределе редуцируются в нелинейное уравнение эллиптического типа.

Традиционньй метод обратной задачи не может быть прямо применен и к нелинейным уравнениям эллиптического и параболического типов, обладающим представлением Лакса (эллиптическое уравнение синус-Гордон, $0(3)$-инвариантная модель, уравнение Эрнста и т.д.). Известно, что интегрируемые параболические уравнения редуцируются в линейные при помоши обобшенного преобразования Коула-Хопфа. Для эллиптических уравнений метод обратной задачи требует сушественной модификации при решении краевых задач. Для уравнения синус-Гордон краевые задачи впервые обсуждались независимо для полуплоскости (с нелокальными краевыми условиями) [8] и для цилиндрически-симметричных сингулярных решений (уравнение гиперболический синус-Гордон) [9-11]. В работах $[10,11]$ была найдена эффективная модификация метода обратной задачи для описания вихреподобных решений уравнения синус-Гордон. Вихреподобные конфигурации полей характеризуются сингулярными решениями $u(x, y) \mathrm{c}$ ненулевым целым топологическим зарядом

$$
Q=\frac{1}{2 \pi} \oint_{\gamma} \nabla u d \mathbf{r},
$$

где $\gamma$ - любой контур, окружающий точку сингулярности-центр вихря. Предложенная процедура позволила описать вихри и вихревые диполи с различными топологическими зарядами, которые представляют собой полубесконечные $2 \pi$-кинки или $2 \pi$-кинки конечной длины, соединяюшие центры вихрей. В магнитных системах такие вихри являются элементарными возбуждениями двумерных систем с обменным взаимодействием и анизотропией или магнитным полем в плоскости пленки. Влияние вихрей на статистические свойства этих систем впервые обсуждались в работе [12]. В протяженных джозефсоновских контактах вихреподобными решениями являются магнитные диполи на фоне кноидальной волны (смешанное состояние сверхпроводников) [13].

Сущность модификации метода обратной задачи для решения подобных эллиптических задач состоит в следуюшем. Используя граничные условия по одной из пространственных переменных (например, $y$ ), можно произвести преобразование к данным рассеяния (в каждой полуплоскости), зависящим от спектрального параметра $\lambda$ и переменной $x$. Граничные условия на функцию $u(x, y)$ при $x \rightarrow \pm \infty$ определяют часть данных рассеяния. Дальнейшая сшивка решений при $x=0$ и условие (1) определяют с точностью до произвольной постоянной сингулярные данные рассеяния. Однако использование этой процедуры для краевых задач в полуплоскости приводит к нелинейным интегральным уравнениям для прямой задачи рассеяния [14], так как пара Лакса неизвестна на прямой $x=0$.

В настоящей работе мы обсуждаем применение метода обратной задачи для решения краевых задач для уравнения синус-Гордон и эллиптической решетки Тоды. Статья построена следуюшим образом. В разделе 2 для эллиптического уравнения синус-Гордон методом обратной задачи рассеяния изучаются сингулярные солитоны - спиральные вихри. Спиральные солитоны, впервые исследованные численным методом [15], отличаются от рассматриваемых нами граничными условиями. Сингулярное поведение таких 
солитонов вблизи центра, зависяшее от параметров $\alpha$ (интенсивность точечного источника) и $Q$ (топологический заряд вихря), позволяет, следуя [9-11], найти обший вид данных рассеяния, зависяший от двух произвольных параметров $\rho$ и $\omega$. Однако, в отличие от авторов указанных работ, мы не определяем параметры $\rho, \omega$ из линеаризованного уравнения синус-Гордон вблизи центра вихря. Мы показываем, что при больших значениях спектрального параметра функции Йоста, ассоциированные со спектральной задачей, имеют автомодельный характер. Это позволяет, используя методы, предложенные в работах $[16,17]$, найти точную нелинейную (перенормированную) связь параметров $\rho$, $\omega$ с $\alpha, Q$ и асимптотическое поведение полей спирального вихря на больших расстояниях от его центра. В разделе 3 описана дискретная эллиптическая модель Тоды в полуплоскости, которая является простым обобщением решетки Тоды и редуцируется в нелинейное уравнение эллиптического типа в континуальном пределе. Мы показываем, что для достаточно общих граничных условий краевая задача для такой системы может быть решена методом обратной задачи. Здесь он сводится к нетривиальной задаче решения нелинейных алгебраических уравнений, которая решается в терминах тэта-констант.

\section{2. КРАЕВАЯ ЗАДАЧА ДЛЯ СПИРАЛЬНОГО ВИХРЯ}

В этом разделе мы обсудим решение краевой задачи уравнения синус-Гордон

$$
u_{x x}(x, y)+u(x, y)_{y y}=\sin u(x, y)
$$

для спирального вихря. Его асимптотика вблизи начала координат в полярной системе координат имеет вид

$$
u \rightarrow-\alpha \ln r+Q \varphi+\beta \quad(r \rightarrow 0)
$$

и удовлетворяет линеаризованному уравнению синус-Гордон вблизи начала координат, исключая точку $r=0$. Первое слагаемое описывает точечный источник силы $\alpha$. Для полей $u(r)$, убываюших при $r \rightarrow \infty$, такие сингулярные решения представляют собой солитоноподобный волновой пакет, они численно исследовались в работах $[17,18]$. Второе и третье слагаемые описывают вихрь с топологическим зарядом $Q$. В дальнейшем без потери общности мы полагаем $Q=-1$. Линии постоянных значений $u(x, y)$ являются логарифмическими спиралями, закручиваюшимися около начала координат. Изменение $u(x, y)$ при обходе вокруг центра вихря по часовой стрелке равно $2 \pi Q$, и для $Q=-1$ мы выберем граничные условия в следующем виде:

$$
\begin{gathered}
u \rightarrow 4 \operatorname{arctg} e^{-y} \quad(\bmod 2 \pi), \quad x \rightarrow \infty, \\
u \rightarrow 0 \quad(\bmod 2 \pi), \quad x \rightarrow-\infty, \\
u \rightarrow 0 \quad(\bmod 2 \pi), \quad y \rightarrow \pm \infty .
\end{gathered}
$$

Эллиптическое уравнение синус-Гордон представляется как условие совместности $L_{x}-A_{y}+[L, A]=0$ двух дифференциальных операторов

$$
\begin{array}{ll}
\Phi_{y}=L \Phi, & L=-i q \sigma_{1}+\frac{i \sigma_{3}}{4}\left(\lambda-\frac{\cos u}{\lambda}\right)+\frac{i}{4 \lambda} \sin u \sigma_{2}, \\
\Phi_{x}=A \Phi+\Phi C, & A=-q \sigma_{1}+\frac{\sigma_{3}}{4}\left(\lambda+\frac{\cos u}{\lambda}\right)-\frac{1}{4 \lambda} \sin u \sigma_{2} .
\end{array}
$$


Здесь $\sigma_{\alpha}(\alpha=1,2,3)$ - матрицы Паули, $q=\left(i u_{x}+u_{y}\right) / 4$ и вид произвольной матрицы $C$ выбирается в соответствии с граничными условиями. Найдем отображение потенциалов в данные рассеяния. С этой целью для вешественных значений $\lambda$ определим матрицы Йоста $\Psi, \Phi$ как решения системы со следуюшим асимптотическим поведением:

$$
\begin{aligned}
\Psi & \rightarrow e^{i k_{2} \frac{\sigma_{3}}{2}} \quad(y \rightarrow \infty), \\
\Phi & \rightarrow e^{i k_{2} \frac{\sigma_{3}}{2}} \quad(y \rightarrow-\infty), \\
k_{2} & =\frac{1}{2}\left(\lambda-\frac{1}{\lambda}\right)
\end{aligned}
$$

Используя треугольное представление матриц Йоста, с помощью стандартной процедуры можно показать, что при условии ограниченности интегралов

$$
\int_{y}^{\infty}|q| d y, \quad \int_{y}^{\infty}|\cos u| d y, \quad \int_{y}^{\infty}|\sin u| d y
$$

вектор-функции

$$
\chi_{\alpha 1}(\lambda)=\Psi_{\alpha 1}(\lambda) e^{-\frac{i k_{2}}{2}}, \quad \chi_{\alpha 2}(\lambda)=\Phi_{\alpha 2}(\lambda) e^{\frac{i k_{2}}{2}} \quad(\alpha=1,2)
$$

аналитически продолжаются в верхнюю полуплоскость комплексной переменной $\lambda$, а вектор-функции

$$
\Psi_{\alpha 2}(\lambda) e^{\frac{i k_{2}}{2}}, \quad \Phi_{\alpha 1}(\lambda) e^{-\frac{i k_{2}}{2}}(\alpha=1,2)
$$

- в нижнюю полуплоскость. Отметим, что из представления (7), (8) следуют ограничения на вид этих матриц:

$$
\Psi(-\lambda)=\sigma_{1} \Psi(\lambda) \sigma_{1}, \quad \Phi(-\lambda)=\sigma_{1} \Phi(\lambda) \sigma_{1},
$$

поэтому матрица рассеяния $T(\lambda): \Psi(\lambda)=\Phi(\lambda) T(\lambda, x)$, имеет вид

$$
T(\lambda, x)=\left(\begin{array}{ll}
a(\lambda, x) & b(-\lambda, x) \\
b(\lambda, x) & a(-\lambda, x)
\end{array}\right)
$$

Найдем зависимость матричных элементов от переменной $x$. Асимптотика функций Йоста не зависит от этой переменной при выборе

$$
C=-\frac{k_{1}}{2} \sigma_{3}, \quad k_{1}=\frac{1}{2}\left(\lambda+\lambda^{-1}\right) .
$$

Тогда из уравнений (8) мы получаем

$$
\begin{gathered}
\frac{d}{d x} T(\lambda, x)=[T(\lambda, x), C], \\
a(\lambda, x)=a(\lambda, 0), \quad b(\lambda, x)=b(\lambda, 0) e^{-k_{1} x} .
\end{gathered}
$$


Обсудим ограничения на данные рассеяния, налагаемые граничными условиями при $x \rightarrow \pm \infty$. Прямыми вычислениями мы находим явный вид функций Йоста в этой асимптотике:

$$
\begin{aligned}
\Psi & \rightarrow e^{i k_{2} y \frac{\sigma_{3}}{2}}, \Phi \rightarrow e^{i k_{2} y \frac{\sigma_{3}}{2}} \quad(x \rightarrow-\infty), \\
\left(\Psi_{11}(\lambda), \Psi_{21}(\lambda)\right) & =\left(\lambda+i \cos \frac{u}{2}, \sin \frac{u}{2}\right) e^{\frac{i k_{2} y}{2}}(\lambda+i)^{-1} \quad(x \rightarrow+\infty), \\
\left(\Phi_{11}(\lambda), \Phi_{21}(\lambda)\right) & =\left(\lambda+i \cos \frac{u}{2}, \sin \frac{u}{2}\right) e^{\frac{i k_{2} y}{2}}(\lambda-i)^{-1} \quad(x \rightarrow+\infty) .
\end{aligned}
$$

Отсюда мы находим важные соотношения $b(\lambda,-\infty)=b(\lambda, \infty)=0$ и определяем часть данных рассеяния:

$$
\begin{aligned}
& a(\lambda)= \begin{cases}(\lambda-i)(\lambda+i)^{-1}, & x>0, \\
1, & x<0,\end{cases} \\
& b(\lambda, x)= \begin{cases}0, & x<0, \quad \lambda>0, \\
0, & x>0, \quad \lambda<0 .\end{cases}
\end{aligned}
$$

Причина различного вида данных рассеяния состоит в том, что при $x=0$ оператор $L$ имеет полюс $(L \sim 1 / y)$, аналогичный полюсу в спектральной задаче Захарова-Шабата [18]. Из-за особенности потенциала $q$ в начале координат функции Йоста неоднозначны в плоскости $(x, y)$, и при обходе по замкнутому контуру они умножаются справа на матрицы монодромии. Мы будем рассматривать отдельно случаи $x<0$ и $x>0$ и обозначать дополнительными индексами (0) и (1) полевые переменные, относящиеся соответственно к левой и правой половинам плоскости $(x, y)$. Так, через $\Phi^{(0)}(\lambda)$ мы будем обозначать функцию Йоста $\Phi(\lambda)$ при $x<0$, через $T^{(0)}(\lambda, x)$ - матрицу рассеяния при $x<0$, а $T^{(n)}(\lambda, x)$ при $x=0$ определим как $T^{(0)}(\lambda,-\varepsilon), T^{(1)}(\lambda, \varepsilon)$ при $\varepsilon \rightarrow 0$. Из представлений $(7),(8)$ нетрудно получить важную, согласованную с граничными условиями редукцию (условие вещественности полей $u(x, y)$ )

$$
\begin{gathered}
\Phi^{(0)}(\lambda)=B \Phi^{*(0)}\left(\frac{1}{\lambda^{*}}\right), \quad \Psi(\lambda)=B \Psi^{*}\left(\frac{1}{\lambda^{*}}\right), \\
T^{(0)}(\lambda)=T^{*(0)}\left(\frac{1}{\lambda^{*}}\right), \quad \Phi^{(1)}(\lambda)=-B \Phi^{*(1)}\left(\frac{1}{\lambda^{*}}\right), \quad T^{(1)}(\lambda)=-T^{*(1)}\left(\frac{1}{\lambda^{*}}\right),
\end{gathered}
$$

где

$$
B=\cos \frac{u}{2}-i \sigma_{1} \sin \frac{u}{2}
$$

Заметим, что из явного вида диагональных элементов матрицы рассеяния и асимптотических разложений нетрудно получить интегральные характеристики спирального вихря и показать, в частности, что уравнение синус-Гордон представимо как условие потенциальности $\Delta_{x, x}+\Delta_{y, y}=0$ векторного поля $\vec{\Delta}=\left(u_{x} u_{y}, u_{y}^{2} / 2-u_{x}^{2} / 2+\cos u-1\right)$. 
Уравнения обратной задачи рассеяния могут быть получены традиционным образом [2] с помощью матричной задачи Римана для аналитической функции

$$
\Omega_{\alpha}= \begin{cases}\Phi_{\alpha 1}(\lambda) \frac{e^{-\frac{i k_{2} y}{2}}}{a(-\lambda)}, & \operatorname{Im} \lambda<0, \\ \Psi_{\alpha 1}(\lambda) e^{-\frac{i k_{2} y}{2}}, & \operatorname{Im} \lambda>0 .\end{cases}
$$

При $x>0$ спектральная задача имеет одно связанное состояние - нуль функции $a^{(1)}(\lambda)=$ $\operatorname{det}\left[\Psi_{\alpha 1}(\lambda), \Phi_{\beta 2}(\lambda)\right]$. Не зависяшую от $x$ постоянную $c$ в соотношении $\left.\Psi_{\alpha 1}(\lambda)\right|_{\lambda=i}=$ $\left.c \Phi_{\alpha 2}(\lambda)\right|_{\lambda=i}$ можно найти из явного вида входящих в него функций при $x \rightarrow \infty$ (см. (13), (14)). Уравнения обратной задачи удобно записать для функций

$$
m(\lambda)=\chi_{1}(-\lambda)+\chi_{2}(-\lambda), \quad n(\lambda)=\chi_{1}(-\lambda)-\chi_{2}(-\lambda)
$$

в следующем виде:

$$
\begin{aligned}
& m^{(1)}(\lambda)= 1-\left.2 m^{(1)}(\lambda)\right|_{\lambda=-i} \frac{e^{-y}}{\lambda-i}- \\
&-\frac{1}{2 \pi i} \int_{0}^{\infty} \frac{r^{(1)}\left(\lambda^{\prime}\right) m^{(1)}\left(\lambda^{\prime}\right)}{\lambda+\lambda^{\prime}-i \epsilon} e^{-k_{1}\left(\lambda^{\prime}\right) x-i k_{2}\left(\lambda^{\prime}\right) y} d \lambda^{\prime}, \\
& \epsilon \rightarrow 0, \quad r^{(1)}(\lambda)=\frac{b^{(1)}(\lambda, 0)}{a^{(1)}(\lambda)}, \quad x>0
\end{aligned}
$$

и

$$
\begin{aligned}
m^{(0)}(\lambda) & =1-\frac{1}{2 \pi i} \int_{-\infty}^{0} \frac{r^{(0)}\left(\lambda^{\prime}\right) m^{(0)}\left(\lambda^{\prime}\right)}{\lambda+\lambda^{\prime}-i \epsilon} e^{-k_{1}\left(\lambda^{\prime}\right) x-i k_{2}\left(\lambda^{\prime}\right) y} d \lambda^{\prime} \\
\epsilon & \rightarrow 0, \quad r^{(0)}(\lambda)=\frac{b^{(0)}(\lambda, 0)}{a^{(0)}(\lambda)}, \quad x<0 .
\end{aligned}
$$

Уравнения для функций $n^{(1)}(\lambda), n^{(0)}(\lambda)$ совпадают с (20), (21) после замены $m^{(1)}(\lambda) \rightarrow n^{(1)}(\lambda), m^{(0)}(\lambda) \rightarrow n^{(0)}(\lambda)$ и смены знака перед интегралом и полюсным слагаемым. Вычисляя коэффициенты асимптотического разложения $\chi_{\alpha}(\lambda)$ по прямым и обратным степеням $\lambda$, из уравнений (7) мы находим выражение полей через функции спектральной задачи:

$$
\begin{gathered}
\chi_{2}(\lambda)=-\frac{2 q}{\lambda}+O\left(\frac{1}{\lambda^{2}}\right), \\
\left.m(\lambda)\right|_{\lambda=0}=e^{-\frac{i u}{2}},\left.\quad n(\lambda)\right|_{\lambda=0}=e^{\frac{i u}{2}}
\end{gathered}
$$

согласованные с уравнениями обратной задачи рассеяния и редукцией

$$
m(\lambda)=m^{*}\left(\frac{1}{\lambda^{*}}\right) e^{-\frac{i u}{2}}, \quad n(\lambda)=n^{*}\left(\frac{1}{\lambda^{*}}\right) e^{\frac{i u}{2}}
$$


являющейся следствием (17), (18).

Отметим важное обстоятельство. Из уравнений (20), (21) следуют замечательные свойства спектральных задач уравнения (2): согласно условию ограниченности потенциалов (ограничения на $b(\lambda, 0))$ функции Йоста допускают аналитическое продолжение с полуплоскости на всю комплексную плоскость с разрезом вдоль луча $(\operatorname{Re} \lambda>0$, $\operatorname{Im} \lambda=0)$ или луча $(\operatorname{Re} \lambda<0, \operatorname{Im} \lambda=0)$.

При $x<0$ мы выберем данные рассеяния в виде

$$
a^{(0)}(\lambda)=1, \quad b^{(0)}(\lambda, 0)=\rho+\omega(\lambda-i)(\lambda+i)^{-1}
$$

по следуюшим причинам. Выражение (25) при вешественном $\rho$ и мнимом $\omega$ - это простейшая рациональная функция, которая дает особенность (3) при итерационном решении уравнений обратной задачи (21) уже в первом порядке. При $\omega=0 \quad(\rho=0)$ данные (25) описывают кольцевую область [17], при $\rho=0$ - вихрь $[10,11]$.

При $x>0$ данные рассеяния выбираются из условия сшивки потенциала при $x=0$ следующим образом. Как следует из граничных условий $(9)$, функции $m(\lambda), n(\lambda)$ непрерывны при $y>0$ на оси $x=0$ и разрывны при $y<0$. Тогда условие непрерывности полей $u(-\epsilon, y)=u(\epsilon, y) \quad(y>0, \varepsilon \rightarrow 0)$ посредством уравнений $(20),(21)$ сводится к интегральным соотношениям, которые в первом итерационном порядке имеют решение $r^{(1)}(\lambda)=-r^{(0)}(\lambda)$. Такое соотношение было справедливо для всех рассматриваемых ранее сингулярных солитонов.

Выразим постоянные $\rho, \omega$ в данных рассеяния через $\alpha, Q$. С этой целью мы используем эффективный прием, предложенный в работах $[16,17]$ при описании сингулярных автомодельных решений $u(r)$ в методе изомонодромных деформаций. Подставим в уравнения (7), (8) асимптотику (3) и определим главные члены асимптотики функций Йоста. Для наших целей достаточно найти их для малых и больших значений $\lambda$. Нетрудно показать, что при $|\lambda|>r^{-1 / 2}$ в уравнениях (7), (8) члены, пропорциональные $\lambda^{-1}$, можно опустить, и упрошенную систему уравнений удобно записать в новых независимых переменных: $r, \gamma=\lambda r \exp (i \varphi)$. Тогда функции $\chi(-\lambda)$ удовлетворяют укороченной системе уравнений

$$
\partial_{\gamma} \chi(-\lambda)=\left(\frac{\sigma_{1}(i \alpha-Q)}{4 \gamma}+\frac{I-\sigma_{3}}{4}\right) \chi(-\lambda)
$$

( $I$ - единичная матрица), общее решение которой выражается через функции Бесселя мнимого аргумента:

$$
\begin{aligned}
& m(\gamma)=e^{\gamma / 4} \sqrt{\gamma}\left[C_{1} I_{\nu}\left(\frac{\gamma}{4}\right)+C_{2} K_{\nu}\left(\frac{\gamma}{4}\right)\right] \\
& n(\gamma)=e^{\gamma / 4} \sqrt{\gamma}\left[-C_{1} I_{\nu+1}\left(\frac{\gamma}{4}\right)+C_{2} K_{\nu+1}\left(\frac{\gamma}{4}\right)\right],
\end{aligned}
$$

с порядком $\nu=(i \alpha-Q) / 4-1 / 2$. С помощью системы уравнений, полученной из $(7),(8)$ и определяюшей зависимость $\chi(-\lambda)$ от переменной $r$, можно показать, что величины $C_{1}, C_{2}$ зависят только от спектрального параметра $\lambda$. При $x<0 \quad(-3 \pi / 2<\varphi<\pi / 2)$ 
функции $m(\lambda), n(\lambda)$ аналитичны в нижней $\lambda$-полуплоскости и в этой области $-\pi / 2<$ $\arg \gamma<3 \pi / 2$. Поэтому из условия нормировки $m(\lambda) \rightarrow 1(\lambda \rightarrow \infty, \operatorname{Im} \lambda<0)$ мы находим $C_{1} \rightarrow O(1 / \lambda), C_{2} \rightarrow 1 / \sqrt{2 \pi}+O(1 / \lambda)$. Аналогичным образом можно определить вид функций Йоста при малых значениях $|\lambda|<r^{1 / 2}$, согласованный с редукциями.

Таким образом, при больших значениях $\lambda$, как и для изомонодромных решений $(Q=0)$, функции Йоста автомодельны (зависят только от переменной $\gamma)$, так как определяются одинаковым типом сингулярности $q=(-i \alpha+Q) \exp (-i \varphi) / 4 r \quad(r \rightarrow 0)$. Поучительно получить вид функции $m(\lambda)$ при больших значениях $\lambda$ из уравнений обратной задачи. Используя редукцию (25) и деформируя контур из интегрального уравнения (21), можно получить замкнутое интегральное уравнение для полей $m(\gamma)(\gamma>r)$. Оно включает интегрирование функций $m(\gamma), m^{*}(\gamma)$ по переменной $\gamma$ только в пределах от $r$ до $\infty$, и в низшем порядке по переменной $r$ имеет простой вид

$$
m(\gamma)=1+\frac{\rho+\omega}{2 \pi i} \int_{0}^{\infty} \frac{m\left(\gamma^{\prime}\right)}{\gamma+\gamma^{\prime}-i \sin g x} e^{-\frac{\gamma^{\prime}}{2}} d \gamma^{\prime}
$$

После замены $m(2 \gamma)=\mu(\gamma) \exp [\gamma / 2]$ интегральное уравнение для полей $\mu(\gamma)$ имеет симметричное ядро

$$
\left(\frac{\rho+\omega}{2 \pi i}\right)\left(\gamma^{\prime}+\gamma\right)^{-1} e^{-\frac{\gamma^{\prime}+\gamma}{2}}
$$

Поскольку функции $K_{\nu}(\gamma / 2) / \sqrt{\gamma}$ являются собственными функциями этого ядра:

$$
\int_{0}^{\infty} e^{-\frac{\gamma^{\prime}+\gamma}{2}} \frac{K_{\nu}\left(\gamma^{\prime} / 2\right)}{\sqrt{\gamma^{\prime}}\left(\gamma^{\prime}+\gamma\right)} d \gamma^{\prime}=\frac{\pi K_{\nu}(\gamma / 2)}{\sqrt{\gamma} / \cos \pi \nu}
$$

то удобно использовать преобразование Лебедева-Конторовича [19]

$$
\mu(\gamma)=\int_{0}^{\infty} K_{i t}\left(\frac{\gamma}{2}\right) \frac{\bar{\mu}(t) d t}{\sqrt{\gamma}}
$$

Тогда непосредственно из интегрального уравнения (29) находим функцию $\bar{\mu}(t)=$ $2 t \operatorname{sh} \pi t / \sqrt{\pi}(\operatorname{ch} \pi t+\cos \pi \nu)$, обратное преобразование которой приводит к соотношению

$$
m(\gamma)=e^{\gamma / 4} \sqrt{\gamma} K_{\nu}\left(\frac{\gamma}{4}\right) \frac{1}{\sqrt{2 \pi}}
$$

где порядок функции Макдональда определяется формулой $\rho+\omega=-2 i \cos \pi \nu$. Сравнивая его со значением, полученным из решения прямой задачи, мы получаем важное соотношение

$$
\rho+\omega=-2 i \sin \left[\frac{\pi(i \alpha-Q)}{4}\right],
$$

связываюшее параметры данных рассеяния с параметрами асимптотического поведения полей $u(x, y)$ вблизи центра спирального вихря. При $Q=0$ соотношение $(31)$ совпадает с полученными в работе [17], при $\alpha=0$ - с результатами работ $[10,11]$, но при малых 
значениях $Q$, так как в этих работах параметр $\omega$ вычислялся некорректно из линейного приближения. Случаю безотражательного потенциала отвечают значения $Q= \pm 4$, $\alpha=0$, при которых вихри можно описать в аналитическом виде [20].

Отметим, что соотношение (31) можно получить из явного вида функций Йоста прямой задачи. Действительно, с учетом области аналитичности и определения полей $m^{(0)}(\lambda)$ уравнение $(21)$ представимо в виде

$$
\begin{aligned}
& m^{(0)}(\lambda)-m^{(0)}\left(\lambda e^{-2 \pi i}\right)=-r^{(0)}(-\lambda) \times \\
& \quad \times m^{(0)}\left(\lambda e^{-\pi i}\right) e^{k_{1}(\lambda) x+i k_{2}(\lambda) y} \quad(\operatorname{Re} \lambda>0),
\end{aligned}
$$

тогда (31) сразу следует из (30) и формул преобразования функций Бесселя.

Обсудим асимптотику спирального вихря при $r \rightarrow \infty$ в наиболее интересной области $x>0$. После поворота контура интегрирования на угол $\varphi$ интеграл в уравнении (20) преобразуется в интеграл по переменной $\tau=\lambda^{\prime} \exp (i \varphi) \quad(0<\tau<1)$ и интересуюшая нас зависимость от $r$ содержится только в множителе $\exp \left[-\left(\tau+\tau^{-1}\right) r / 2\right]$. Тогда асимптотика решения уравнения (20) при $r \rightarrow \infty$ вычисляется методом Лапласа и основной вклад дает точка $\tau=1$. Вычисляя таким образом функцию $m^{(1)}(\lambda)$ и ее значение при $\lambda=0$, мы находим асимптотическую формулу при $r \rightarrow \infty$ и конечных значениях $x$ для полей $u$ в первом порядке:

$$
u \rightarrow u_{0}+\frac{\cos \left(\varphi-u_{0} / 2\right)}{\cos \varphi} e^{-r} \sqrt{\frac{2}{\pi r}}\left[\rho-i \omega \operatorname{tg}\left(-\frac{\pi}{4}+\frac{\varphi}{2}\right)\right],
$$

где $u_{0}=4 \operatorname{arctg}[\exp (-y)]$. Отсюда следует, что в этом порядке на больших расстояниях солитон деформируется и центр солитона $(u=\pi)$ не сдвигается. Аналогичным образом можно найти громоздкую формулу для полей во втором порядке и показать, что при $\alpha>0$ центр солитона сдвигается на величину

$$
\Delta y=\frac{\sin (\pi \alpha / 4) e^{-r}}{\sqrt{\pi} r^{3 / 2}}+O\left(e^{-2 r}\right)
$$

\section{3. КРАЕВАЯ ЗАДАЧА ДЛЯ ЭЛЛИПТИЧЕСКОЙ РЕШЕТКИ ТОДЫ}

Эллиптическая цепочка Тоды

$$
-Q_{n, x x}=e^{-\left(Q_{n}-Q_{n-1}\right)}-e^{-\left(Q_{n+1}-Q_{n}\right)}
$$

описывает деформацию в анизотропных кристаллах в случае, если взаимодействие между цепочками атомов (индекс $n$ ) нелинейно и континуальное приближение не применимо. Уравнение (33) допускает представление Лакса

$$
\begin{aligned}
(L \varphi)_{n} & \equiv i\left[a_{n-1} \varphi_{n-1}+a_{n} \varphi_{n+1}\right]+b_{n} \varphi_{n}=\lambda \varphi_{n} \\
\frac{d \varphi_{n}}{d x} & =i\left[a_{n-1} \varphi_{n-1}-a_{n} \varphi_{n+1}\right] .
\end{aligned}
$$


Здесь введены обозначения

$$
b_{n}=\frac{1}{2} Q_{n, x}, \quad a_{n}=\frac{1}{2} e^{-\frac{1}{2}\left(Q_{n+1}-Q_{n}\right)},
$$

в терминах которых уравнение (33) имеет простой вид:

$$
a_{n, x}=a_{n}\left(b_{n}-b_{n-1}\right), \quad b_{n, x}=2\left(a_{n}^{2}-a_{n-1}^{2}\right) .
$$

Мы обсудим краевую задачу Дирихле для уравнений (36) с периодическими граничными условиями по дискретному индексу

$$
a_{n+N}=a_{n}, \quad b_{n+N}=b_{n},
$$

краевыми условиями по переменной $x(0<x<\infty)$

$$
Q_{n} \rightarrow 0, \quad x \rightarrow \infty
$$

и заданными смешениями $g_{n}$ на границе $x=0$

$$
\left.Q_{n}(x)\right|_{x=0}=g_{n} .
$$

Процедура решения краевой задачи (36)-(39) состоит в следуюшем.

Преж де всего, используя граничные условия (37), мы отобразим потенциалы $a_{n}, b_{n}$ в данные рассеяния: спектр и дополнительный спектр оператора $L$. С этой целью согласно [21] вводятся два фундаментальных решения $S(n), C(n)$ уравнения (34) с граничными условиями $C(0)=S(1)=1, C(1)=S(0)=0$. Тогда след $\Lambda(\lambda)=C(N)+S(N+1)$ матрицы монодромии является интегралом движения $(d \Lambda / d x=0)$ системы (35). Дополнительный спектр $\mu_{i}(k)(i=1,2, \ldots, N-1)$ определим корнями функций $C(h, \lambda \mid k)$, $\left.C(N+1, \lambda \mid k)\right|_{\lambda=\mu_{i}(k)}=0$. Здесь $C(h, \lambda \mid k)$ - решения уравнения (34) (в котором сделана замена $\left.a_{n} \rightarrow a_{n+k}, b_{n} \rightarrow b_{n+k}\right)$ с граничными условиями $C(0, \lambda \mid k)=1, C(1, \lambda \mid k)=0$. Эволюция переменных $\mu_{k}$ согласно (35) имеет вид

$$
\mu_{k, x}=\left(\frac{i}{2}\right)^{N} \frac{ \pm 2 \sqrt{\Delta^{2}\left(\mu_{k}\right)-4}}{\prod_{j \neq k}\left(\mu_{k}-\mu_{j}\right)} .
$$

Далее с помошью граничных условий при $x \rightarrow \infty$ мы находим функцию $\Lambda(\lambda)=z^{N}+$ $z^{-N}$, где $\lambda=(i / 2)\left(z+z^{-1}\right)$, и, следовательно, спектр $z_{k}=\exp (i \pi k / N), k=0, \pm 1, \ldots$ $\ldots, \pm N$, оператора $L$. Отметим, что этот оператор не определен при $x=0$ и традиционный метод прямой и обратной задач не применим. В нашем случае при $x=0$ необходимо решить смешанную задачу: по известной функции $\Lambda(\lambda)$ и потенциалам $\left.a_{n}(x)\right|_{x=0}=g_{n}$ найти другие потенциалы $\left.b_{n}(x)\right|_{x=0}$ и дополнительный спектр $\left.\mu_{k}(x)\right|_{x=0}$. 
Примечательно, что явный вид дискриминанта Хилла определяет численное значение интегралов движения. Так при $N=3$ мы находим следуюшие значения интегралов движения:

$$
\begin{gathered}
I_{1}=b_{1} b_{2}+b_{3}=0 \\
I_{2}=b_{1} b_{2}+b_{2} b_{3}+b_{1} b_{3}+a_{1}^{2}+a_{2}^{2}+a_{3}^{2}=\frac{3}{4} \\
I_{3}=b_{1} b_{2} b_{3}+b_{1} a_{2}^{2}+b_{2} a_{3}^{3}+b_{3} a_{1}^{2}=0
\end{gathered}
$$

и решение краевой задачи в этом случае, в отличие от задач классической механики, есть решение системы (36) с заданными значениями интегралов движения и обобщенными координатами $\left.a_{n}(x)\right|_{x=0}$.

Найдем сначала общее решение задачи. Несложные вычисления показывают, что функции Блоха $\varphi^{ \pm}(x, n) \quad\left(\varphi^{ \pm}(n+N)=z^{ \pm N} \varphi^{ \pm}(n)\right)$ мероморфны в плоскости комплексной переменной $z\left(\lambda=(i / 2)\left(z+z^{-1}\right)\right)$ и имеют следуюший вид:

$$
\varphi^{ \pm}(k+1, z)=z^{ \pm k} \prod_{i=1}^{N-1}\left(\frac{z-\nu_{i}^{ \pm 1}(k)}{z-\nu_{i}^{ \pm 1}}\right) e^{ \pm\left(\frac{Q_{k+1}-Q_{1}}{2}\right)}
$$

где $\nu_{i}=\nu_{i}(k=0)$ и $\mu_{k}=(i / 2)\left(\nu_{k}+\nu_{k}^{-1}\right)$. Наконец, используя соотношения между $\nu_{i}(k)$ и $\nu_{i}[21]$

$$
\sum_{i=1}^{N-1} \int_{\nu_{i}}^{\nu_{i}(k)} \omega_{l}(z) d z=\int_{z_{l}^{-1}}^{z_{l}}\left(\chi^{+}(k)-\frac{k}{z}\right) d z=-2 k \ln z_{l}
$$

и

$$
\frac{d}{d x} \sum_{i=1}^{N-1} \int_{\nu_{0}}^{\nu_{i}(k)} \omega_{l}(\nu) d \nu=i
$$

где

$$
\omega_{l}(z)=\left(z-z_{l}\right)^{-1}\left(z-z_{l}^{-1}\right)^{-1}, \quad \chi(k)=\frac{d}{d z} \ln \varphi^{+}(k+1),
$$

мы находим зависимость от $x$ симметризованных функций от $\nu_{i}(k)$ в явном виде:

$$
\prod_{i=1}^{N-1} \frac{\nu_{i}(k)-z_{l}}{\nu_{i}(k)-z_{l}^{-1}}=z^{-2 k} e^{2 t_{l} x} C_{l}, \quad l=1,2, \ldots, N-1, \quad t_{l}=\frac{i}{2}\left(z_{l}-z_{l}^{-1}\right) .
$$

Здесь выполняются редукции $C_{l}=C_{N-l}^{*}$ и $\nu_{i}^{*}=-\nu_{N-i}$.

В результате обшее решение системы (36) с краевыми условиями (37), (38) имеет простой вид

$$
e^{Q_{k}}=\frac{D(k-1)}{D(k)}
$$

где $D(k)=\operatorname{det} A$ и элементы квадратной $((N-1) \times(N-1))$-матрицы $A$

$$
A_{l s}(k)=\left(-z_{l}\right)^{s-1} e^{-t_{l} x}-\left(-z_{l-1}\right)^{-s+1}\left(z_{l}\right)^{-2 k} C_{l} e^{t_{l} x}
$$


зависят от постоянных $C_{l}$.

Таким образом, решение краевой задачи (36)-(39) редуцируется в решение системы нелинейных алгебраических уравнений

$$
e^{g_{k}}=\left.\frac{D(k-1)}{D(k)}\right|_{x=0},
$$

определяюших постоянные $C_{l}$ через заданные переменные $g_{n}$. Детерминанты, входяшие в формулу (42), являются аналогами тэта-функций [22] и линейны по любой постоянной $C_{l k}=\left.z_{l}^{-2 k} C_{l}\left(z_{l}\right)\right|_{z_{l}=\exp [i \pi l / N]}$ :

$$
\begin{aligned}
& D(k)(-1)^{N-1}=W\left(z_{1}, z_{2}, \ldots, z_{N-1}\right)-\sum_{i} C_{i k} W\left(z_{1}, z_{2}, \ldots, z_{i}^{-1}, \ldots, z_{N-1}\right)+ \\
& \quad+\sum_{i<j} C_{i k} C_{j k} W\left(z_{1}, z_{2}, \ldots, z_{i}^{-1}, \ldots, z_{j}^{-1}, \ldots, z_{N-1}\right)- \\
& \quad-\sum_{i<j<l} C_{i k} C_{j k} C_{l k} W\left(z_{1}, z_{2}, \ldots, z_{i}^{-1}, \ldots, z_{j}^{-1}, \ldots, z_{l}^{-1}, \ldots, z_{N-1}\right)-\cdots
\end{aligned}
$$

где $W$ - определитель Вандермонда. Мы нашли, что при $N=3$ постоянные $C_{1}, C_{2}=C_{1}^{*}$ определяются уравнением четвертого порядка. При $N=4$ соответствуюшая система уравнений редуцируется в нелинейные уравнения по каждой переменной $C_{1}, C_{2}, C_{3}=$ $C_{1}^{*}$, степень которых выше пятой. Численные расчеты показывают, что эти уравнения не имеют кратных корней, поэтому, за исключением специальных случаев, явное решение уравнений (42) возможно только в терминах тэта-констант (см. в [22] Х. Умемура "Решение алгебраических уравнений с помощью тэта-констант").

Итак, мы показали, что решение краевой задачи совместно с методом обратной задачи рассеяния и редушируется в решение системы алгебраических уравнений. Их эффективная “линеаризация" будет обсуждаться нами в другой работе. В заключение заметим, что в терминах тэта-функций возможно получить общее решение и для ограниченной эллиптической решетки Тоды $(0<x<l)$. В этом случае краевая задача при заданных смешениях $\left.Q_{n}(x)\right|_{x=0},\left.Q_{n}(x)\right|_{x=l}$ редуцируется в нетривиальную задачу определения параметров тэта-функций.

Благодарности. Автор глубоко признателен В.В. Дякину за интерес к работе и консультации по интегральным уравнениям и С. А. Зыкову за помощь в проведении численных расчетов. Работа выполнена при финансовой поддержке РФФИ (грант № 97-0100431).

\section{Список литературы}

[1] М. Абловии, Х. Сигур. Солитоны и метод обратной задачи рассеяния. М: Мир, 1987.

[2] В.Е. Захаров, С.В. Манаков, С.П. Новиков, Л.П. Питаевский. Теория солитонов: Метод обратной задачи. М.: Наука, 1980.

[3] A.S. Fokas. Physica D. 1989. V. 35. P. 167.

[4] E.K. Sklyanin. J. Phys. A. 1988. V. 21. P. 2375.

[5] А. С. Фокас, А.Р. Итс. ТМФ. 1992. Т. 92. № 3. С. 387. 
[6] R.F. Bikbaev, V. O. Tarasov. J. Phys. A. 1991. V. 24. P. 2507.

[7] И. Т. Хабибуллин. ТМФ. 1992. Т. 91. № 3. С. 363.

[8] Е. С. Гутшабаш, В. Д. Липовский. Зап. научн. семин. ЛОМИ. 1990. Т. 180. С. 53.

[9] D. J. Kaup. Two new aspects of the inverse scattering transform: (1) the elliptic sinh-Gordon equation, and (2) growing solitons. In: Nonlinear World. Proceed. 4th Intern. Wookshop on Nonlinear and Turbulent Processes in Physics (Kiev, 1989). V. 1. Ed. V.G. Bar'yakhtar. River Edge, NJ: World Scientific, 1990. P. 139.

[10] A.B. Borisov, S. N. Ionov, A.G. Shagalov. New types of vortices and the solutions of the boundary value problem for the sine-Gordon equation by the inverse scattering transform. In: Nonlinear World. Proceed. 4th Intern. Wookshop on Nonlinear and Turbulent Processes in Physics (Kiev, 1989). V. 1. Ed. V. G. Bar'yakhtar. River Edge, NJ: World Scientific, 1990. P. 65.

[11] A. B. Borisov. Phys. Lett. A. 1990. V. 143. № 1. P. 52.

[12] M.D. Einhorn, R, Savit, E. A. Rabinovich. Nucl. Phys. 1980. V. 170. P. 16.

[13] A. B. Borisov, V. V. Kiseliev. Physica D. 1995. V. 87. P. 64.

[14] A.B. Borisov, S.N. Ionov, A.G. Shagalov. Vortices, strings and the solutions of the boundary value problem by the inverse scattering transform. In: Proceed. NEED's 92. Eds. V. G. Makhan'kov, F. Calogero. Singapore: World Scientific, 1992. P. 67.

[15] A. G. Shagalov. Phys. Lett. A. 1992. V. 165. P. 412.

[16] A.R. Its, V.Yu. Novokshenov. The Isomonodromic Deformation in the Theory of the Painleve' Equations. Lecture Notes in Mathematics. V. 1191. Berlin-Heidelberg-New York: Springer-Verlag, 1986.

[17] В. Ю. Новокшенов, А.Г. Шагалов. ТМФ. 1997. Т. 111. С. 15.

[18] Ю. И. Яльшев. Частное сообщение, 1994.

[19] В. А. Аркадьев, А.К. Погребков, М. К. Поливанов. ТМФ. 1982. Т. 53. С. 163.

[20] В. А. Диткин, А. П. Прудников. Интегральные преобразования и операционное исчисление. М.: Физматгиз, 1961.

[21] М. Тода. Теория нелинейных решеток. М.: Мир, 1984.

[22] Д. Мамфорд. Лекции о тэта-функциях. М.: Мир, 1988.

Поступила в редакцию 22.XII.1999 г. 